\title{
Chiral Symmetry Breaking from Center Vortices
}

\section{Roman Höllwieser*}

Institute of Atomic and Subatomic Physics, Vienna University of Technology, Austria and Department of Physics, New Mexico State University, Las Cruces, NM 88003-8001, USA

E-mail: hroman@kph.tuwien.ac.at

\section{Manfried Faber}

Institute of Atomic and Subatomic Physics, Vienna University of Technology, Austria

E-mail: faber@kph.tuwien.ac.at

\section{Thomas Schweigler}

Institute of Atomic and Subatomic Physics, Vienna University of Technology, Austria

\section{Urs M. Heller}

American Physical Society, One Research Road, Ridge, NY 11961, USA

E-mail: hellereaps.org

We analyze the creation of near-zero modes from would-be zero modes of various topological charge contributions from classical center vortices in $S U(2)$ lattice gauge theory. We show that colorful spherical vortex and instanton configurations have very similar Dirac eigenmodes and also vortex intersections are able to give rise to a finite density of near-zero modes, leading to chiral symmetry breaking via the Banks-Casher formula. We discuss the influence of the magnetic vortex fluxes on quarks and how center vortices may break chiral symmetry. ${ }^{\dagger \dagger}$

31st International Symposium on Lattice Field Theory - LATTICE 2013

July 29 - August 3, 2013

Mainz, Germany

\footnotetext{
* Speaker.

${ }^{\dagger}$ This research was partially supported by the Austrian Science Fund (FWF) under contract P22270-N16 (R.H.).

\$ The numerical calculations were performed at the Vienna Scientific Cluster (VSC).
} 


\section{Introduction}

A well established theory of spontaneous chiral symmetry breaking $(\chi \mathrm{SB})$ relies on instantons, which are localized in space-time and carry a topological charge of modulus 1 . According to the Atiyah-Singer index theorem, a zero mode of the Dirac operator arises, which is concentrated at the instanton core. In the instanton liquid model overlapping would-be zero modes split into lowlying nonzero modes which create the chiral condensate. Center vortices are promising candidates for explaining confinement. The vortex model of confinement is theoretically appealing and was confirmed by a multitude of numerical calculations, both in lattice Yang-Mills theory and within a corresponding infrared effective model, see e.g. [1,2]. Lattice simulations indicate that vortices are responsible for topological charge and $\chi \mathrm{SB}$ as well [3, 4, 5], and thus unify all nonperturbative phenomena in a common framework. A similar picture to the instanton liquid model exists insofar as lumps of topological charge arise at the intersection and writhing points of vortices. The colorful, spherical $S U(2)$ vortex was introduced in a previous article of our group [6] and may act as a prototype for this picture, as it contributes to the topological charge by its color structure, attracting a zero mode like an instanton. We show how the interplay of various topological structures from center vortices (and instantons) leads to near-zero modes, which by the Banks-Casher relation are responsible for a finite chiral condensate.

\section{Free Dirac eigenmodes}

The chiral density of free overlap eigenmodes obtained numerically using the MILC code are shown in Fig. 1. The modes are found with the Ritz functional algorithm with random start and for degenerate eigenvalues the eigenmodes span a randomly oriented basis in the degenerate subspace. Therefore the numerical modes presented in Fig. 1 are linear combinations of plane waves with momenta $\pm p_{\mu}$ and show plane wave oscillations of $2 p_{\mu}$ in the chiral density. For $12^{3} \times 24$ lattices, the first eight degenerate modes consist of plane waves with $p_{4}= \pm \pi / 24$, hence there is one sine (cosine) oscillation in time direction, the next eight have $p_{4}= \pm 3 \pi / 24$, i.e., three oscillations in the time direction. The oscillations of $\chi_{R}$ and $\chi_{L}$ are separated by half an oscillation length, i.e., the maxima of $\rho_{+}$correspond to minima of $\rho_{-}$and vice versa, accordingly, the scalar density is constant as expected for free eigenmodes.
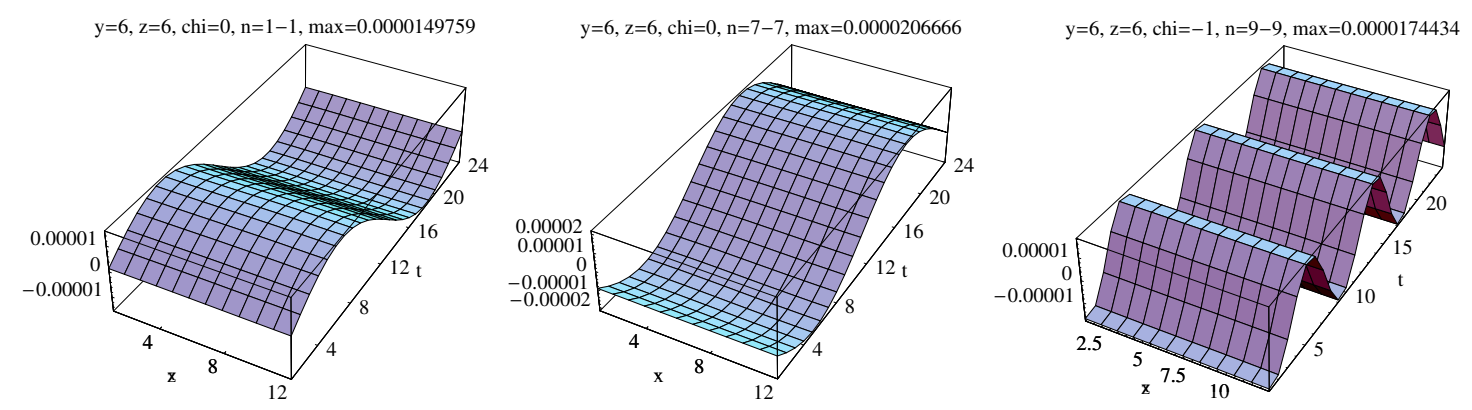

Figure 1: Chiral density of the low-lying eigenmodes of the free overlap Dirac operator: $\rho_{5} \# 1$ (left), $\rho_{5} \# 7$ (center) $\rho_{5} \# 9$ (right). The modes clearly show the plane wave behavior with oscillations of $2 p_{\mu}$ (see text). 


\section{The Colorful Spherical Vortex}

The spherical vortex was introduced in [6] and analyzed in more detail in [7, 8, 9]. It is constructed with $t$-links in a single time slice at fixed $t=t_{i}$, given by $U_{t}\left(x^{v}\right)=\exp \left(\mathrm{i} \alpha\left(\left|\vec{r}-\vec{r}_{0}\right|\right) \vec{r} / r \cdot \vec{\sigma}\right)$, where $\vec{r}$ is the spatial part of $x_{v}$. The profile function $\alpha(r)$ changes from $\pi$ to 0 in radial direction for the negative spherical vortex, or from $\pi$ to $2 \pi$ for the positive (anti-)vortex. This gives a hedgehog-like configuration, since the color vector points in (or against) the radial direction at the vortex radius $R$. The hedgehog-like structure is crucial for our analysis. The $t$-links of the spherical vortex define a map $S^{3} \rightarrow S U(2)$, characterized by a winding number $N=-1$ for positive (anti-) and $N=+1$ for negative spherical vortices. Obviously such windings influence the Atiyah-Singer index theorem giving a topological charge $Q=-1$ for positive and $Q=+1$ for negative spherical vortices (anti-vortices) and attract Dirac zero modes similar to instantons. In [9] we showed that the spherical vortex is in fact a vacuum-to-vacuum transition in the time direction which can even be regularized to give the correct topological charge also from gluonic definitions. Fig. 2a shows that a spherical vortex has nearly exactly the same eigenvalues as an instanton. We further plot the spectra of instanton-anti-instanton, spherical vortex-anti-vortex and instanton-anti-vortex pairs. We again see nearly exactly the same eigenvalues for instanton or spherical vortex pairs, instead of two would-be zero modes there is a pair of near-zero modes for each pair. The chiral density plots in Fig. 3 for the instanton-anti-instanton pair and Fig. 4 for the spherical vortex-anti-vortex pair show, besides the similar densities, that the near-zero modes are a result of two chiral parts corresponding to the two constituents of the pairs. The nonzero modes can be identified with the free overlap modes, as they show plane-wave behavior. In Fig. $2 \mathrm{~b}$ we plot the eigenvalues of two (anti-)instantons and two spherical (anti-)vortices giving topological charge $Q=2(Q=-2)$ and therefore two zero modes, two vortex-anti-vortex pairs with two pairs of near-zero modes and a configuration with two vortices and an anti-vortex (i.e., a single vortex plus one vortex-anti-vortex pair) giving one zero mode $(Q=1)$ and one pair of near-zero modes. The results show that we may draw the same conclusions for spherical vortices as for instantons concerning the creation of near-zero modes.

a)

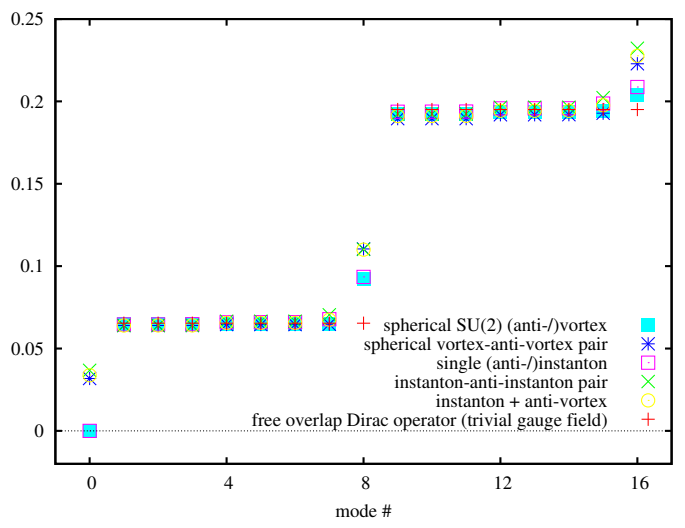

b)

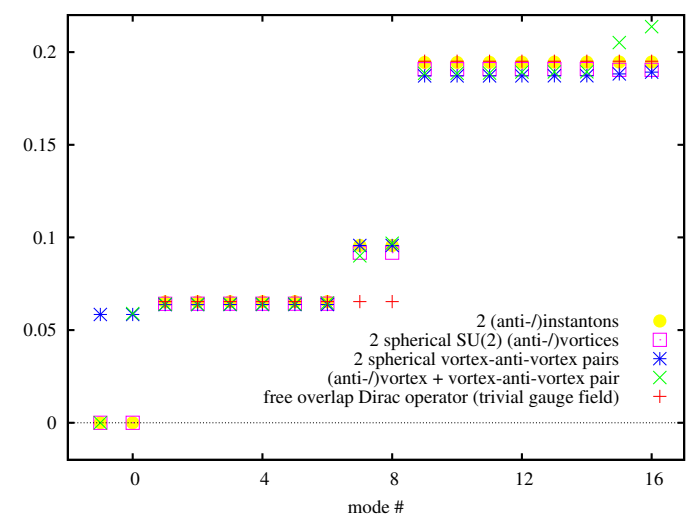

Figure 2: The lowest overlap eigenvalues for instanton and spherical vortex configurations compared to the eigenvalues of the free (overlap) Dirac operator. 
a)
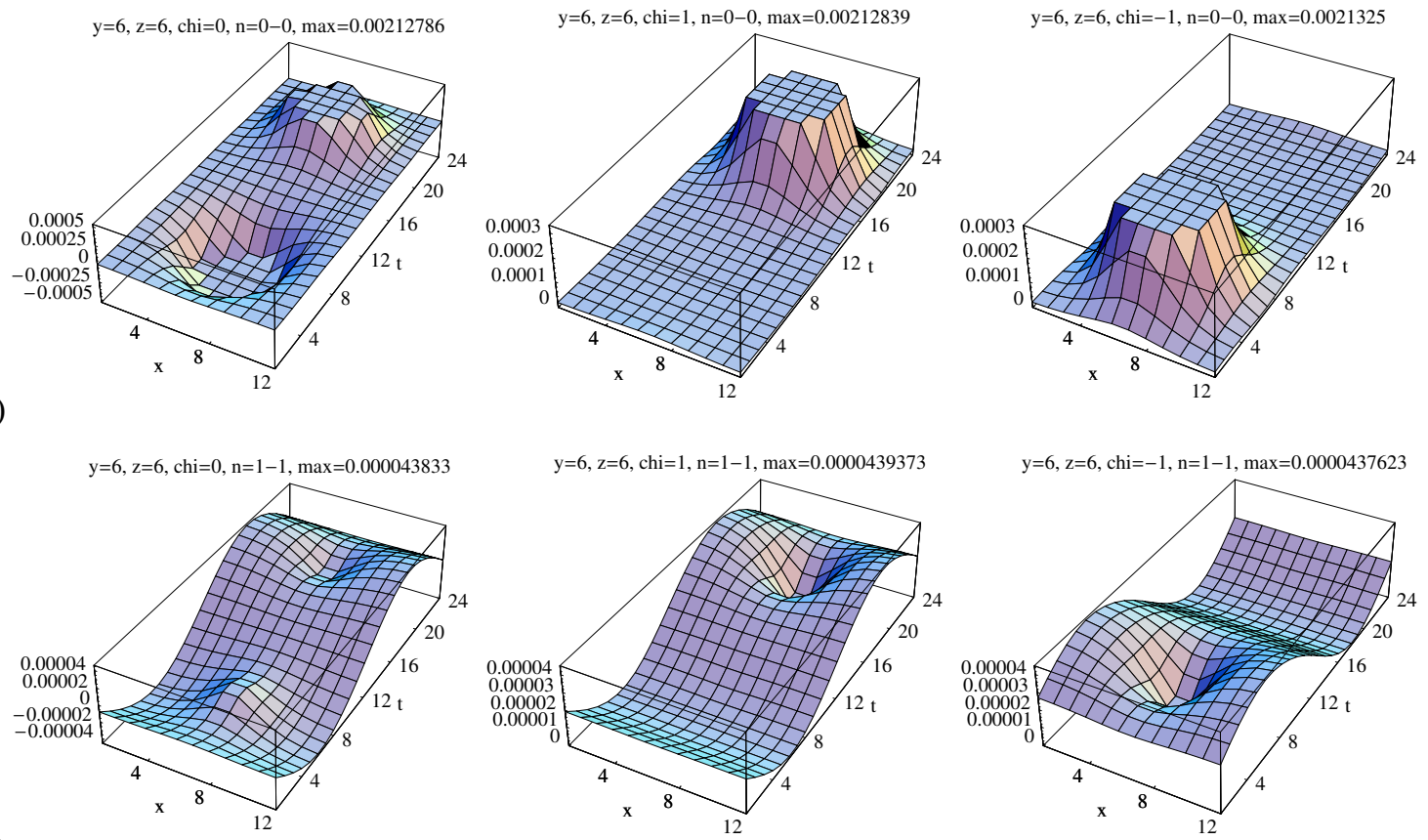

b)

$y=6, z=6$, chi $=0, n=8-8, \max =0.00105352$ $\mathrm{y}=6, \mathrm{z}=6, \mathrm{chi}=1, \mathrm{n}=8-8, \max =0.00105532$

$\mathrm{y}=6, \mathrm{z}=6, \mathrm{chi}=-1, \mathrm{n}=8-8, \max =0.00105369$
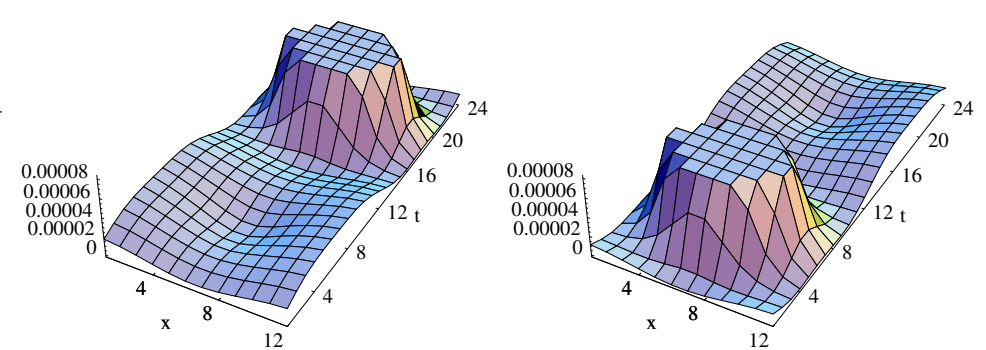

c)

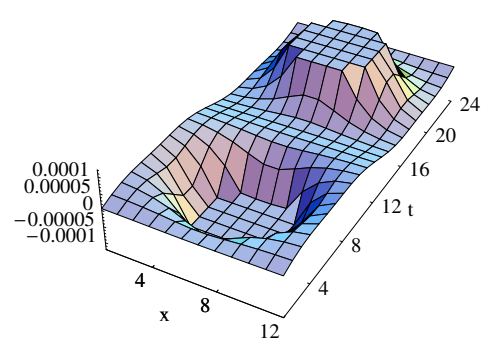

$y=6, z=6$, chi $=0, n=7-7, \max =0.000065997$

$y=6, z=6$, chi $=0, n=9-9, \max =0.0000617173$
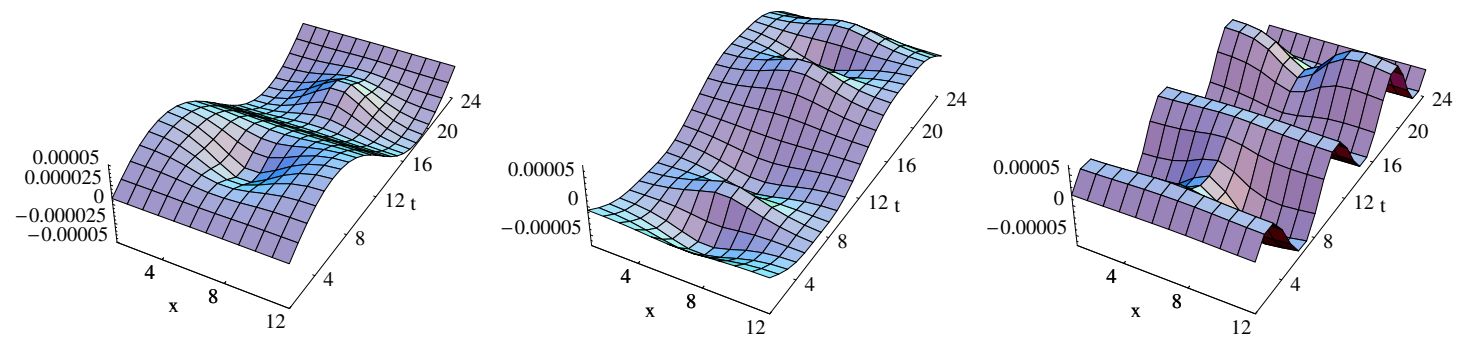

d)

Figure 3: Chiral densities ( $\rho_{5}$ left, $\rho_{+}$center and $\rho_{-}$right column) of the a) lowest (near-zero), b) secondlowest (nonzero) and c) eighth (nonzero) eigenmode of the overlap Dirac operator for an instanton-antiinstanton pair. d) $\rho_{5}$ of the sixth (left), seventh (center) and ninth (right) eigenmode. 

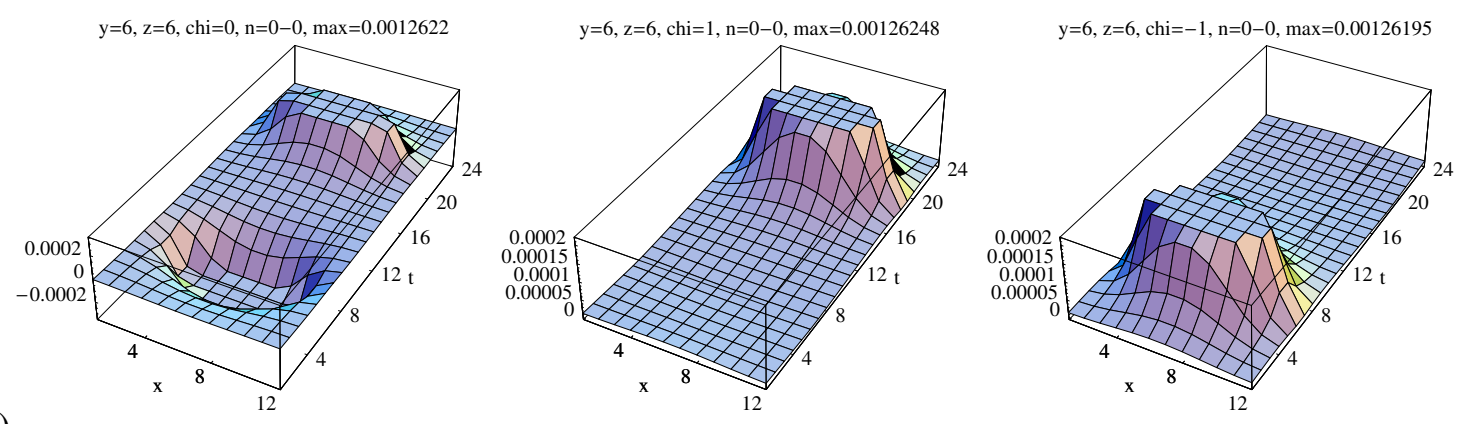

a)

$\mathrm{y}=6, \mathrm{z}=6, \mathrm{chi}=0, \mathrm{n}=1-1, \max =0.000055155$

$\mathrm{y}=6, \mathrm{z}=6, \mathrm{chi}=1, \mathrm{n}=1-1, \max =0.0000553718$

$\mathrm{y}=6, \mathrm{z}=6, \mathrm{chi}=-1, \mathrm{n}=1-1, \max =0.0000553037$
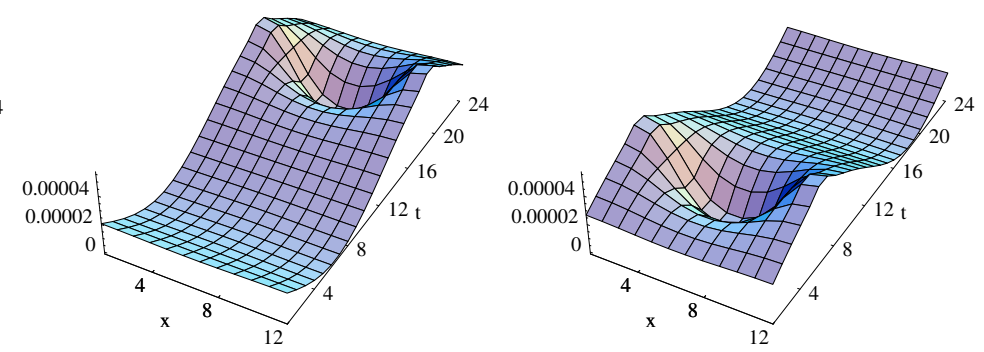

b)

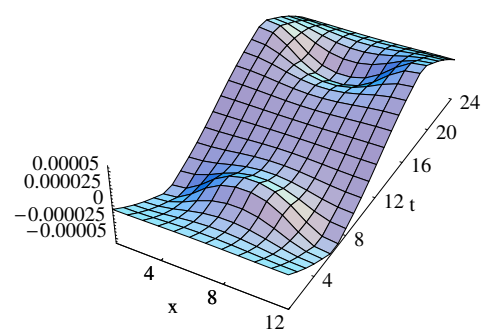

$\mathrm{y}=6, \mathrm{z}=6, \mathrm{chi}=1, \mathrm{n}=8-8, \max =0.000563865$

$\mathrm{y}=6, \mathrm{z}=6, \mathrm{chi}=-1, \mathrm{n}=8-8, \max =0.000564226$
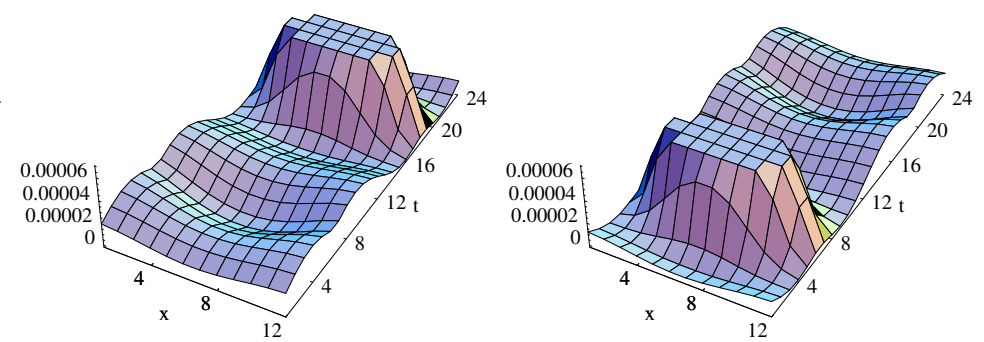

c)

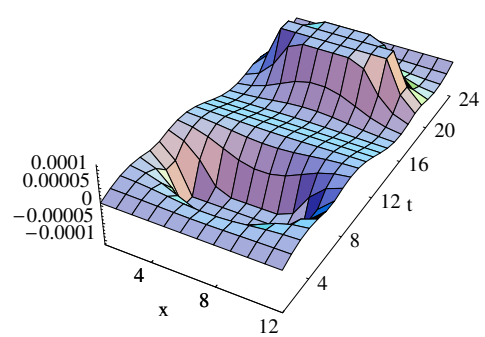

$y=6, z=6, \operatorname{chi}=0, n=7-7, \max =0.0000650816$

$\mathrm{y}=6, \mathrm{z}=6, \mathrm{chi}=-1, \mathrm{n}=9-9, \max =0.0000998798$
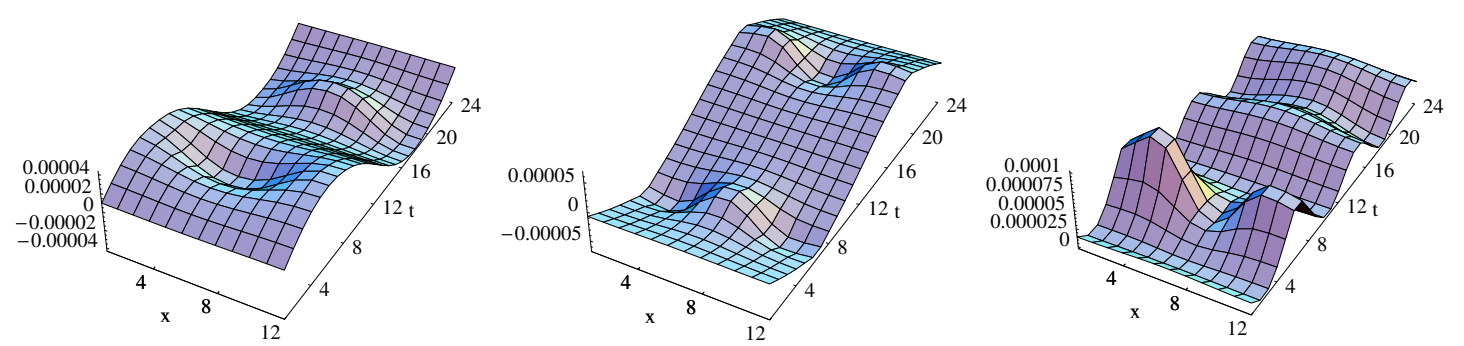

d)

Figure 4: Same as Fig. 3 but for a spherical vortex-anti-vortex pair. Chiral densities ( $\rho_{5}$ left, $\rho_{+}$center and $\rho_{-}$right column) of the a) lowest (near-zero), b) second-lowest (nonzero) and c) eighth (nonzero) eigenmode. d) $\rho_{5}$ of the sixth (left), seventh (center) and ninth (right) eigenmode. 


\section{Plane vortices}

We define plane vortices parallel to two of the coordinate axes by links varying in a $U(1)$ subgroup of $S U(2)$. This $U(1)$ subgroup is generated by one of the Pauli matrices $\sigma_{i}$, i.e., $U_{\mu}=$ $\exp \left(\mathrm{i} \phi \sigma_{i}\right)$. Upon traversing a vortex sheet, the angle $\phi$ increases or decreases by $\pi$ within a finite thickness of the vortex. Since we use periodic (untwisted) boundary conditions for the links, vortices occur in pairs of parallel sheets, each of which is closed by virtue of the lattice periodicity. We call vortex pairs with the same vortex orientation parallel vortices and vortex pairs of opposite flux direction anti-parallel. If thick, planar vortices intersect orthogonally, each intersection carries a topological charge $|Q|=1 / 2$, whose sign depends on the relative orientation of the vortex fluxes [10]. For intersecting parallel vortices we get two real zero modes, according to the total topological charge $Q=2$ of the four intersections. These modes we analyzed in [11], they peak at least at two of the four topological charge contributions of $Q=1 / 2$. If we intersect anti-parallel vortex pairs orthogonally we get two intersection points with topological charge $Q=+1 / 2$ and two intersection points with topological charge $Q=-1 / 2$, hence total $Q=0$. For such a configuration we get four real near-zero modes, with local chirality peaks at the intersection points, according to their topological charge contribution, see Fig. 5b. The mechanism of Sec. 3 or the analog instanton liquid model does not directly apply to the case of planar vortices, since there are no localized lumps of topological charge $Q= \pm 1$. But vortex intersections with topological charge $Q= \pm 1 / 2$ might be related to merons and calorons. In any case, we showed that they, too, can create a finite density of near-zero modes and thus cause $\chi \mathrm{SB}$ via the Banks-Casher relation.

a)

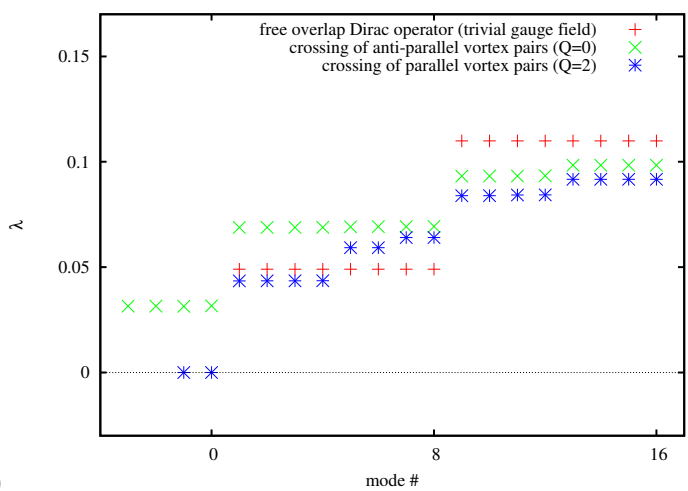

b)

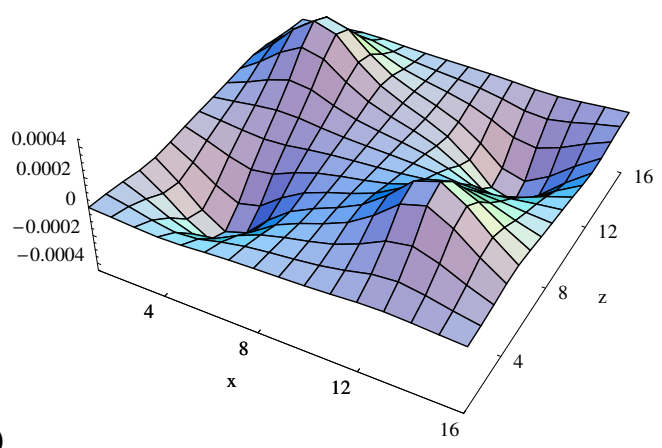

Figure 5: a) The lowest overlap eigenvalues for plane vortex configurations compared to the eigenvalues of the free (overlap) Dirac operator (red crosses) and spherical vortex configurations. b) Chiral density in the intersection plane of all four near-zero modes of crossing flat vortex pairs with opposite flux direction $(Q=0)$.

\section{Conclusions}

Fermions do not seem to make much of a difference between instantons and spherical vortices and the instanton liquid model can be extended to colorful spherical center vortices. Further also vortex intersections attract (would-be) zero modes which contribute via interactions to a finite density of near-zero modes with local chiral properties, i.e., local chirality peaks at corresponding topological charge contributions. In Monte Carlo configurations we do not, of course, find perfectly 
flat or spherical vortices, as one does not find perfect instantons. The general picture of topological charge from vortex intersections, writhing points and even color structure contributions can provide a general picture of $\chi \mathrm{SB}$ : just like instantons any source of topological charge can attract (wouldbe) zero modes and produce a finite density of near-zero modes leading to chiral symmetry breaking via the Banks-Casher relation. Here one also has to ask what could be the dynamical explanation of $\chi \mathrm{SB}$. We can try the conjecture that only a combination of color electric and magnetic fields leads to $\chi \mathrm{SB}$, electric fields accelerating color charges and magnetic fields trying permanently to reverse the momentum directions on spiral shaped paths. Such reversals of momentum keeping the spin of the particles should especially happen for very slowly moving color charges. Alternatively we could argue that magnetic color charges are able to flip the spin of slow quarks, i.e. when they interact long enough with the vortex structures. Finally, it seems that vortices not only confine quarks into bound states but also break chiral symmetry. For more details see [12].

\section{References}

[1] L. Del Debbio, M. Faber, J. Greensite, and Š. Olejník, "Center dominance and Z(2) vortices in SU(2) lattice gauge theory," Phys. Rev. D 55 (1997) 2298-2306, arXiv: 9610005 [hep-lat ] .

[2] M. Engelhardt and H. Reinhardt, "Center vortex model for the infrared sector of Yang-Mills theory: Confinement and deconfinement," Nucl. Phys. B585 (2000) 591-613, arXiv: 9912003 [hep-lat].

[3] P. de Forcrand and M. D'Elia, "On the relevance of center vortices to QCD," Phys. Rev. Lett. 82 (1999) 4582-4585, arXiv: 9901020 [hep-lat ] .

[4] M. Engelhardt, "Center vortex model for the infrared sector of Yang-Mills theory: Quenched Dirac spectrum and chiral condensate," Nucl.Phys. B638 (2002) 81-110, arXiv:0204002 [hep-lat] .

[5] R. Höllwieser, M. Faber, J. Greensite, U.M. Heller, and Š. Olejník, "Center Vortices and the Dirac Spectrum," Phys. Rev. D 78 (2008) 054508, arXiv:0805.1846 [hep-lat] .

[6] G. Jordan, R. Höllwieser, M. Faber, U.M. Heller, "Tests of the lattice index theorem," Phys. Rev. D 77 (2008) 014515, arXiv:0710.5445 [hep-lat].

[7] R. Höllwieser, M. Faber, U.M. Heller, "Lattice Index Theorem and Fractional Topological Charge," arXiv:1005.1015 [hep-lat].

[8] R. Höllwieser, M. Faber, U.M. Heller, "Critical analysis of topological charge determination in the background of center vortices in SU(2) lattice gauge theory," Phys. Rev. D 86 (2012) 014513, arXiv:1202.0929 [hep-lat].

[9] T. Schweigler, R. Höllwieser, M. Faber, and U.M. Heller, "Colorful SU(2) center vortices in the continuum and on the lattice," Phys. Rev. D 87 (2013) 054504, arXiv:1212.3737 [hep-lat] .

[10] M. Engelhardt and H. Reinhardt, "Center projection vortices in continuum Yang-Mills theory," Nucl. Phys. B567 (2000) 249, arXiv: 9907139 [hep-th].

[11] R. Höllwieser, M. Faber, U.M. Heller, "Intersections of thick Center Vortices, Dirac Eigenmodes and Fractional Topological Charge in SU(2) Lattice Gauge Theory," JHEP 1106 (2011) 052, arXiv:1103.2669 [hep-lat].

[12] R. Höllwieser, T. Schweigler, M. Faber and U.M. Heller, "Center Vortices and Chiral Symmetry Breaking in SU(2) Lattice Gauge Theory," submitted to Phys.Rev.D (2013), arXiv:1304.1277 [hep-lat]. 